\title{
Freiwilligkeit als Ressource
}

\section{Die Engagementlandschaft im Paritätischen Wohlfahrtsverband - Beispiel Thüringen}

\section{ANDREAS KOTTER}

Andreas Kotter ist Referent des Stiftungsvorstandes der "PARITÄTISCHEN BuntStiftung Thüringen«. Einer der Schwerpunkte der Stiftung ist das bürgerliche Engagement. In diesem Zusammenhang ist Andreas Kotter "Engagementverantwortlicher" und im "Steuerkreis Engagement« zuständig für das Zusammenspiel mit den anderen Unternehmen des Paritätischen Wohlfahrtsverbandes in Thüringen. www.buntstiftung.de

\author{
Bedeutsam für die Gewinnung Ehrenamtlicher ist es \\ für eine Organisation, je eigene Strategien zum Erhalt \\ Freiwilliger zu entwickeln, neue Engagementformen zu \\ etablieren, freiwillig Engagierte in das Unternehmen \\ zu integrieren, zu qualifizieren und Engagement \\ mit hauptamtlichen Strukturen zu verbinden, \\ wie eine Studie am Beispiel Thüringen zeigt.
}

In den vergangenen Jahren hat das Thema Engagement in Deutschland, aber natürlich auch im Freistaat Thüringen fachlich und gesellschaftlich an Bedeutung gewonnen. Dem Paritätischen Wohlfahrtsverband kommt dabei seit jeher eine besondere Aufmerksamkeit zu. Die Vielfalt des Engagements in seinen Mitgliedsorganisationen, Einrichtungen und Diensten erstreckt sich durch alle Facetten freiwilligen und bürgerschaftlichen Engagements. Zudem ist der Paritätische Dachorganisation von Selbsthilfegruppen und Selbsthilfeinitiativen.

Gemeinnützige Unternehmen der Sozialwirtschaft sind u. a. geprägt durch ein hohes freiwilliges Engagement, das sich in der direkten Arbeit mit hilfebedürftigen Menschen, aber auch in der Unternehmensführung durch Vorstände spiegelt. Freiwilliges Engagement ist ein wesentliches Merkmal Paritätischer Mitgliedsorganisationen. Dabei verlangen Strukturveränderungen in Unternehmen und Gesellschaft neue Modelle und Formen der koordinierten Einbindung freiwillig Engagierter in Unternehmen der Sozialwirtschaft.

Entscheidend für ein erfolgreiches und wettbewerbsfähiges Angebot ist, eigene Strategien zum Erhalt freiwillig Engagierter zu entwickeln, neue Engagementformen zu etablieren, freiwillig Engagierte in das Unternehmen zu integrieren, zu qualifizieren und Engagement mit hauptamtlichen Strukturen zu verbinden.

Im Mittelpunkt der Anstrengungen, Engagierte zu gewinnen und zu hal- ten, stehen Themen aus den Bereichen Organisationsentwicklung und Mitarbeiterqualifizierung. Erfahrungen aus dem Bundesfreiwilligendienst können eingeschlagene Wege bestärken, indem dadurch Engagierte langfristig in die Vereinsarbeit eingebunden werden. Entscheidend sind jedoch für ein Gelingen die Rahmenbedingungen der Freiwilligen-und Ehrenamtsarbeit, wozu auch Schlagworte wie Anerkennungskultur und die Symbiose von Haupt- und Ehrenamt gehören.

\section{Potenziale untersucht}

Um den aktuellen Stellenwert und die Bedeutung des Engagements im Paritätischen Wohlfahrtsverband umfassend zu erheben und zu analysieren, wurde vom Paritätischen Thüringen in Kooperation mit dem Paritätischen Gesamtverband in Berlin, den Landesverbänden Berlin und Nordrhein-Westfalen und der MartinLuther-Universität Halle-Wittenberg sowie der Carl von Ossietzky Universität Oldenburg eine grundlegende empirische Untersuchung des Engagements und seiner Potenziale in den Mitgliedsorganisationen, Diensten und Einrichtungen des Paritätischen Wohlfahrtsverbands durchgeführt.

Von 335 Mitgliedsorganisationen (Stand 2011) haben 166 Mitgliedsorganisationen anonym an der Befragung teilgenommen. (1) Allein für diese 166 Mitgliedsorganisationen wurden 5.341 Engagierte gezählt. 80 Prozent davon 
sind im operativen Geschäft tätig, 20 Prozent im Management (Gremien). 19 Prozent der Engagierten sind in der Selbsthilfe tätig. Dies entspricht der Tradition der Mitgliedsorganisationen des Paritätischen. Jedoch haben gerade diese Selbsthilfegruppen mit der Nachfolgeproblematik zu kämpfen. Sie suchen Engagierte, die sich sowohl in dem Themengebiet engagieren, aber auch in Gremien und Netzwerken stark machen. Hier sind eine gezielte Ansprache und möglicherweise auch gezieltere Qualifizierungsangebote ratsam.

\section{Junge und alte Menschen weniger engagiert als erwartet}

Die Altersstruktur der Engagierten (2), wie sie von den Mitgliedsorganisationen angegeben wurden, hinterlässt den Eindruck eines Engagierten-Stammes von 38 Prozent in der Altersspanne 46 bis 65 Jahre. Der Anteil der Engagierten zwischen 31 und 45 Jahren ist nahezu gleich groß wie der von Engagierten zwischen 66 und 79 Jahren. Gerade bei den oft zitierten »engagierten Senioren « liegt der Anteil niedriger als erwartet, anders als beispielsweise im Landesverband Berlin, wo der Anteil der über 65-Jährigen bei 40 Prozent liegt. Engagierte bis 19 Jahre sowie zwischen 20 und 30 Jahre treten in Thüringen nur zu 8 bzw. 11 Prozent auf. Vor allem diese Altersgruppe der »engagierten Jungen « sollte aus Sicht des Paritätischen Thüringen ist eine wesentliche Personengruppe, um langfristig den personellen Unterbau der Vereinsarbeit zu erhalten und so auch bei der Vorstandstätigkeit Perspektiven zu erhalten.

Um »engagierte Junge« zu gewinnen, braucht es möglicherweise andere Formen der Ansprache, aber auch Möglichkeiten sich zu engagieren. Es sollte keinesfalls zu einem Gegeneinander von »Alteingesessenen « und "jungen Wilden« kommen, beide Gruppen haben ihre Berechtigung und sollten ihren Raum im Vereinsleben bekommen. Vermeintliche Parallelstrukturen können sich möglicherweise durchaus positiv für die Vereinsentwicklung auswirken, wenn dies als Chance zur Nutzung von Synergieeffekten genutzt werden kann. Fühlen sich neue Engagierte wohl in den Einrichtungen, kann über eine Art Mentorenprogramm versucht werden, Perspektiven in Richtung Vorstandstätigkeit einzuschlagen.
Entsprechend des Länderreports Thüringen werden die Engagierten in den befragten Paritätischen Mitgliedsorganisationen in Thüringen hauptsächlich im Kinder- und Jugendbereich, aber auch in der Bildungsarbeit eingesetzt. Wie erwartet, sind ehrenamtlich Engagierte in der Behindertenhilfe und im Selbsthilfebereich aktiv, zwei starke Bereiche im Paritätischen Thüringen. Bei der Hilfe für Menschen in besonderen sozialen Situationen, bei der Flüchtlingshilfe sowie in der Suchthilfe sind jedoch wenige Engagierte anzutreffen. (3) Oftmals sind dafür jedoch vermeintliche Berührungsängste, eigene Vorbehalte und mangelndes Fachwissen die Gründe.

Die Tätigkeitsschwerpunkte für Engagierte sind der Befragung zufolge vor allem die Organisation von Veranstaltungen, Interessenvertretung sowie praktische Arbeit und Öffentlichkeitsarbeit. Vor allem für das Erscheinungsbild nach außen, wozu Veranstaltungen und Öffentlichkeitsarbeit meist dienen, werden also Engagierte oft benötigt. Die Gremienarbeit lebt vor allem von ehrenamtlich Engagierten, die sich in den (Fach-) Gremien meist vor Ort mit ihrer Erfahrung einbringen. (4)

Als Gründe für den Einsatz Engagierter wurden seitens der Geschäftsführungen und der Engagementverantwortlichen in den Einrichtungen vor allem die hohe Motivation der Engagierten und die Stärkung des sozialen Miteinanders vor Ort angegeben. Aber auch die Nutzung der Erfahrung von Engagierten und die Tradition der Organisationen, die immer mit Engagierten gearbeitet haben, werden als wichtige Gründe genannt. (5) Werden ehrenamtlich Engagierte gut angeleitet und haben die Möglichkeit, ihren Platz in den Einrichtungen einzunehmen und sich einzubringen, können gerade sie die Hefe im Teig erfolgreicher Organisationsentwicklung sein.

Die umfassende empirische Untersuchung des Engagements in der Studie »Engagement in der Freien Wohlfahrtspflege « hat den Verband mit seinen Unternehmen herausgefordert und wird ganz nach der Devise »vor der Studie ist nach der Studie « herausfordern. Die Engagementstudie des Paritätischen und vor allem der Länderbericht Thüringen haben Befunde geliefert, zu deren Umsetzung derzeit intensiv in verschiedenen Formaten die Strategie besprochen wird, sowohl innerhalb der Paritätischen
Unternehmen in Thüringen, im Steuerkreis Engagement als dann auch mit den Mitgliedsorganisationen. Geeignete Formate, um mit den Mitgliedsorganisationen in den Diskurs zu kommen, werden derzeit erarbeitet.

\section{Nachhaltige Kooperationen zwischen Haupt- und Ehrenamt gesucht}

Mit Blick auf die demografische Entwicklung wird die nachhaltige Verbindung von Haupt- und Ehrenamt aus unserer Sicht in der Zukunft massiv notwendig. Familiäre Strukturen lösen sich auf. Zur Kompensation dieser Entwicklung ist ehrenamtliches Engagement eine Ressource im additiven Sinne. Jedoch darf ehrenamtliches Engagement in den Paritätischen Einrichtungen kein »Nice to have" werden, sondern ein "Must have«.

Engagement sollte als Chefsache in den Einrichtungen verstanden werden, als ein Puzzleteil auf dem Weg, unsere Mitgliedsorganisationen zukunftsfest zu machen.

\section{Anmerkungen}

(1) Backhaus-Maul, H., Speck, K. et al.; 2014; S. 407.

(2) Backhaus-Maul, H., Speck, K. et al.; 2014; S. 408.

(3) Backhaus-Maul, H., Speck, K. et al.; 2014; S. 400.

(4) Backhaus-Maul, H.; Speck, K. et al.; 2014; S. 410.

(5) Backhaus-Maul, H.; Speck, K. et al.; 2014; S. 419.

\section{Literatur}

Backhaus-Maul, Holger; Speck, Karsten et al: Engagement in der Freien Wohlfahrtspflege - Engagementpotenziale und Rahmenbedingungen in den Mitgliedsorganisationen, Einrichtungen und Diensten des Paritätischen Wohlfahrtsverbandes. Ein Projektbericht; Halle (Saale); 2014 